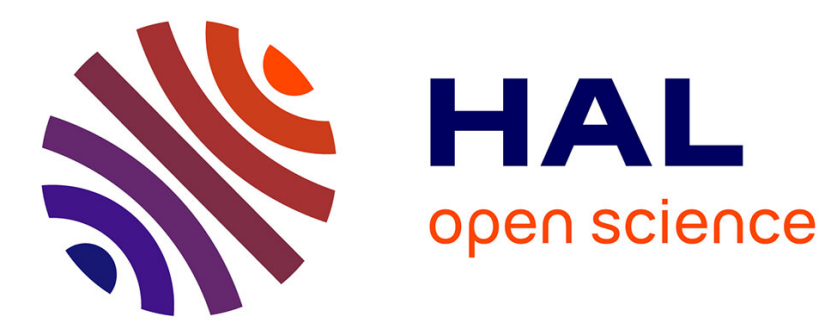

\title{
Réalisation pratique d'une discrimination neutron-gamma
}

\author{
J. Kahane, R. Sené, P. Delpierre, M. Heyman
}

\section{To cite this version:}

J. Kahane, R. Sené, P. Delpierre, M. Heyman. Réalisation pratique d'une discrimination neutrongamma. Revue de Physique Appliquée, 1969, 4 (2), pp.257-258. 10.1051/rphysap:0196900402025700 . jpa-00243249

\section{HAL Id: jpa-00243249 https://hal.science/jpa-00243249}

Submitted on 1 Jan 1969

HAL is a multi-disciplinary open access archive for the deposit and dissemination of scientific research documents, whether they are published or not. The documents may come from teaching and research institutions in France or abroad, or from public or private research centers.
L'archive ouverte pluridisciplinaire HAL, est destinée au dépôt et à la diffusion de documents scientifiques de niveau recherche, publiés ou non, émanant des établissements d'enseignement et de recherche français ou étrangers, des laboratoires publics ou privés. 


\title{
RÉALISATION PRATIQUE D’UNE DISGRIMINATION NEUTRON-GAMMA
}

\author{
J. KAHANE, R. SENÉ, P. DELPIERRE et M. HEYMAN, \\ Laboratoire de Physique Atomique et Moléculaire, Collège de France.
}

\begin{abstract}
Résumé. - Une méthode simple et pratique de discrimination neutron-gamma est obtenue à partir d'un circuit résonnant $R C L$ placé à une seule dynode de photomultiplicateur. On détecte $99,5 \%$ des neutrons de plus de $500 \mathrm{keV}$, tandis que $99 \%$ des gamma sont rejetés.
\end{abstract}

Abstract. - A simple and convenient method of neutron-gamma discrimination is achieved by a damped $R C L$ circuit attached to a single photomultiplier dynode. $99.5 \%$ of the neutrons above $500 \mathrm{keV}$ are detected while $99 \%$ of the gamma are rejected.

Sans aborder l'aspect théorique du problème, nous avons mis en œuvre un système de discrimination $n-\gamma$ pratique, c'est-à-dire applicable de façon simple à de nombreux détecteurs simultanément, et facile à contrôler périodiquement. Au départ nous désirons caractériser un type de particules ionisantes (les protons de recul) à l'exclusion d'un autre (les électrons d'effet Compton). L'ensemble scintillateur (NE 213)-photomultiplicateur (56 AVP 03) qui forme un détecteur nous fournit déjà deux informations (temps et énergie) prélevées à l'anode; nous utilisons pour l'identification la douzième dynode.

La technique de base est la discrimination de forme des impulsions, d'après le schéma désormais classique du mécanisme de la scintillation dans certains liquides organiques $[1,2]$. Selon cette théorie, la scintillation se décompose, en fonction du temps, en deux fractions : une composante rapide (effet immédiat), à décroissance purement exponentielle (demi-période 3 à $6 \mathrm{~ns}$ ), qui est prépondérante au début mais s'éteint rapidement (50 à $100 \mathrm{~ns}$ ) — une composante lente (effet différé) à décroissance non exponentielle mais de durée beaucoup plus grande que la composante principale, et par conséquent dont l'effet devient sensible après 50 ou 100 ns.

Pour annuler l'effet de la composante rapide, et caractériser la composante lente grâce à un paramètre indépendant de l'énergie incidente, nous nous sommes inspirés du système de détection du passage à zéro de l'impulsion doublement différenciée [3]. C'est ainsi que nous avons placé, à la sortie dynode, un circuit résonnant $R C L$ au voisinage de l'amortissement critique, soit : $R C=T$ et $L C=4 T^{2}$. En fait, nous avons simplement ajouté une self $L$ en parallèle au circuit $R G$ de sortie.

Nous allons brièvement justifier ce choix. En négligeant la dispersion des temps de transit dans le PM, nous pouvons décrire le courant de dynode par la fonction du temps : $I=I_{0}\left[\lambda \mathrm{e}^{-t / \tau}+\mu f(t)\right]$ avec $I_{0}$ proportionnel à l'énergie incidente, $\lambda$ proportion de la composante rapide (à période de décroissance exponentielle $\tau$ ), $\mu$ proportion de la composante lente (à décroissante $f(t)$ non explicitée).

L'hypothèse fondamentale est que les paramètres $\lambda$ et $\mu$ sont indépendants du temps et de l'énergie, et caractérisent le type de particule ionisante. En prenant $T=R C$ grand devant $\tau$ (10 à 20 fois), le calcul approché de l'amplitude $V$ de l'impulsion de dynode aboutit à la forme suivante :

$$
V=V_{0}\left[\lambda\left(1-\frac{t}{2 T}\right) \mathrm{e}^{-t / 2 T}+\mu \varphi(t)\right]
$$

où $V_{0}$ est un facteur proportionnel à l'énergie et $\varphi(t)$ une fonction du temps, compliquée mais ne dépendant pas de l'énergie. Au voisinage de $t=2 T$, l'exponentielle et $\varphi(t)$ varient très lentement et se comportent comme des constantes. Alors $V$ est une fonction linéaire du temps qui s'annule pour une valeur $t=t_{0}$ donnée par l'équation :

$$
\lambda\left(1-\frac{t_{0}}{2 T}\right) \mathrm{e}^{-t_{0} / 2 T}+\mu \varphi\left(t_{0}\right)=0 .
$$

Le passage à zéro de l'impulsion de sortie est ainsi caractérisé par $\lambda$ et $\mu$. Le décalage de $2 T$ à $t_{0}$ est proportionnel à $\mu / \lambda$. Entre deux types de particules ionisantes ayant un rapport $\mu / \lambda$ différent, la séparation est possible, indépendamment de l'énergie incidente.

Dispositif EXPÉRIMENTAL ( $f g .1$ ). - Chaque sortie dynode 12 des PM détecteurs est immédiatement reliée à un intermédiaire qui contient la cellule $R C L$ et deux émetteurs-suiveurs en série servant d'abaisseurs d'impédance et de limiteurs. La sortie du limiteur est une impulsion bipolaire écrêtée à $\pm 0,5 \mathrm{~V}$ et transmise, comme les voies temps et énergie, par un câble coaxial de $100 \Omega$. Les temps de passage à zéro sont les suivants (pour un $2 T=280 \mathrm{~ns}$ ) : passage des gamma : $288 \mathrm{~ns} \pm 3$; passage des neutrons : $308 \mathrm{~ns} \pm 5$. Le décalage à la traversée ( $8 \mathrm{~ns}$ et $28 \mathrm{~ns}$ ) est dû à la contri- 


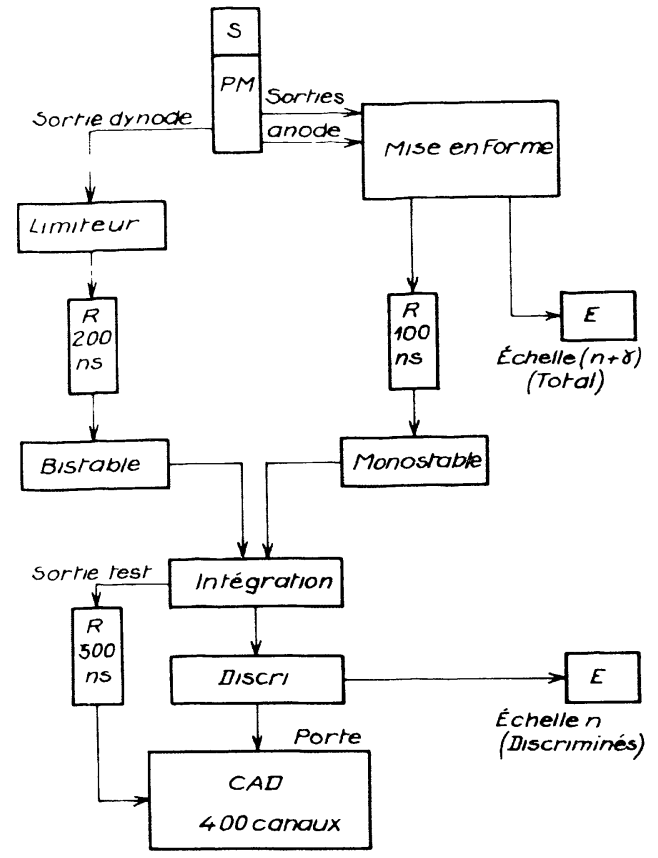

FIG. 1. - Schéma logique de la discrimination de forme.

bution de la composante lente. L'impulsion bipolaire est ensuite traitée dans un tiroir modulaire [4]. Après amplification, l'impulsion bipolaire attaque la voie stop d'un convertisseur temps-amplitude simplifié dont la voie start est attaquée par une impulsion de la voie temps normale. Ainsi l'amplitude de sortie est linéairement fonction du temps de passage à zéro de l'impulsion d'entrée. En fait, les temps de passage sont allongés par rapport à ceux de la sortie du limiteur, ceci étant dû au temps de stockage des divers étages de traitement. L'essentiel reste que la dispersion autour des points de traversée n'augmente pas sensiblement. Le même tiroir modulaire comprend une sortie destinée à la spectrométrie de contrôle et un discriminateur linéaire qui sépare les amplitudes supérieures (neutrons) des inférieures (gamma et bruit de fond).

Performances. - La séparation obtenue est d'autant meilleure que le seuil (voie linéaire) est plus élevé, non seulement à cause de la remontée exponentielle du nombre d'impulsions de basse énergie (non-linéarité du scintillateur), mais aussi des dispersions de temps de transit dans le PM et de la moins bonne définition de la traversée à zéro à faible pente. Si bien que la méthode semble difficilement applicable au-dessous de $100 \mathrm{keV}$ électrons.

Nos résultats usuels ( $f g$. 2), au seuil 1/3 Cs 137 (150 $\mathrm{keV} \mathrm{e}^{-}$ou $500 \mathrm{keV}$ protons) avec un cylindre

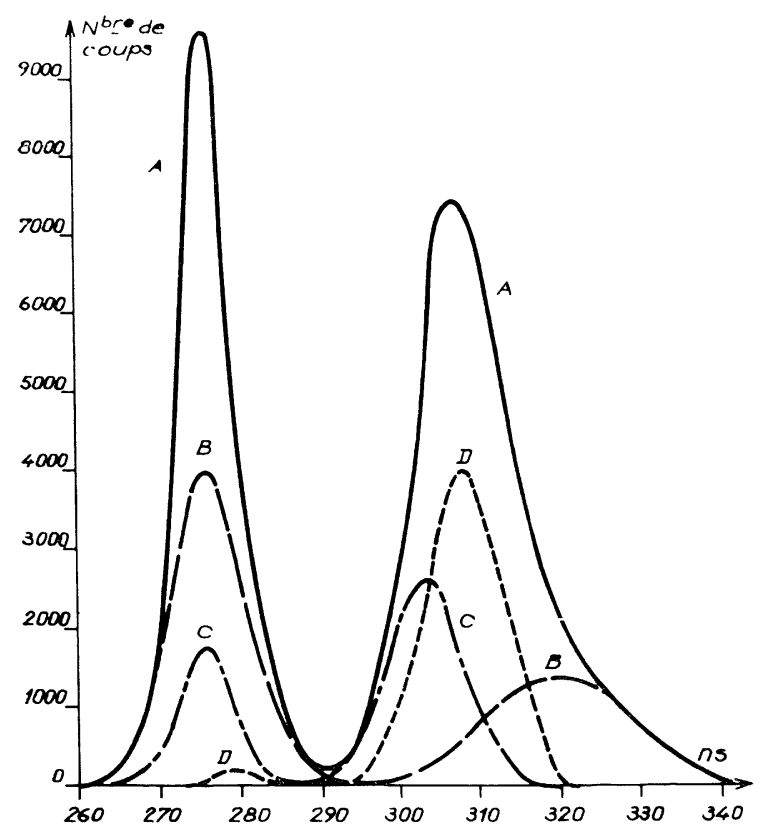

FIG. 2. - Spectres de contrôle de séparation $n-\gamma: A$, spectre toutes énergies ; $\mathrm{B}$, énergies inférieures ; $\mathrm{C}$, énergies moyennes ; D, énergies supérieures.

de $5 \times 5 \mathrm{~cm}$ de NE 213 et un flux de 1000 à 10000 particules détectées par seconde, démontrent que 99,5\% des protons sont conservés et $99 \%$ des gamma sont rejetés. Les protons perdus sont d'énergie inférieure, les gamma comptés sont d'énergie élevée. L'influence du flux moyen devient très sensible au-delà de 10 000/s. En effet, les circuits traitent des impulsions du domaine de la microseconde. Mais en cas de nécessité, on peut travailler jusqu'à 100 000/s.

La séparation $n-\gamma$ par bandes d'énergie fait apparaître la plus grande dispersion lorsque l'énergie est la plus basse. Mais elle montre aussi qu'avec un traitement biparamétrique la séparation serait quasi totale. C'est ce que nous avons en projet pour équiper les détecteurs de nos expériences en neutrons polarisés.

\section{BIBLIOGRAPHIE}

[1] Birks (J. B.), Theory and Practice of Scintillation Counting, Pergamon Press, 1964.

[2] Walter (G.), Méthodes d'identification des particules, colloque de Lyon, 1967.
[3] Alexander (T. K.) et Goulding (F. S.), Nucl. Instr. Methods, 1961, 13, 244.

[4] Kahane (J.), Rapport interne, P.A.M., Collège de France, 1968. 